\title{
EXPONENTIAL COORDINATES AND REGULARITY OF GROUPOID HEAT KERNELS
}

\author{
BING KWAN SO
}

\begin{abstract}
We prove that on an asymptotically Euclidean boundary groupoid, the heat kernel of the Laplacian is a smooth groupoid pseudo-differential operator.
\end{abstract}

\section{INTRODUCTION}

In this notes we study the regularity of certain groupoid heat kernels.

1.1. The problem. We begin with stating our problem, recalling some basic notations along the way. Our motivation will be explained in the next section.

First of all, we briefly recall the notion of geoupoid pseudo-differential operators and heat kernels. The theory is developed by Nistor, Weinstein and $\mathrm{Xu}[8]$ and is considered classical.

Let $\mathcal{G} \rightrightarrows \mathrm{M}$ be a Lie groupoid. We shall assume $\mathcal{G}$ is Hausdorff. Denote the source and the target maps by $\mathbf{s}$ and $\mathbf{t}$ respectively. We shall use the convention $\mathbf{s}(a b)=\mathbf{s}(b), \forall a, b \in \mathcal{G}, \mathbf{t}(b)=\mathbf{s}(a)$. For each $x \in \mathrm{M}$, write $\mathcal{G}_{x}:=\mathbf{s}^{-1}(x)$ for the s-fiber over $x$, and $T^{\perp} \mathcal{G}:=\{X \in T \mathcal{G}: d \mathbf{s}(X)=0\}$. Note that $T^{\perp} \mathcal{G}$ is just the tangent space of all the $\mathcal{G}_{x}$.

Definition 1.1. A pseudo-differential operator $\Psi$ on a groupoid $\mathcal{G}$ of order $\leq m$ is a smooth family of pseudo-differential operators $\left\{\Psi_{x}\right\}_{x \in \mathrm{M}}$, where $\Psi_{x} \in \Psi^{m}\left(\mathcal{G}_{x}\right)$, and satisfies the right invariance property

$$
\Psi_{\mathbf{s}(a)}\left(\mathbf{r}_{a}^{*} f\right)=\mathbf{r}_{g}^{*} \Psi_{\mathbf{t}(a)}(f), \quad \forall a \in \mathcal{G}, f \in C_{c}^{\infty}\left(\mathcal{G}_{\mathbf{s}(a)}\right) .
$$

If, in addition, all $\Psi_{x}$ are classical of order $m$, then we say that $\Psi$ is classical of order $m$.

Definition 1.2. The operator $\Psi$ is called uniformly supported if the set

$$
\left\{a b^{-1}:(a, b) \in \operatorname{Supp}(\Psi)\right\}
$$

is a compact subset of $\mathcal{G}$.

One particularly interesting groupoid differential operator is the Laplacian. Let $\mathrm{A} \rightarrow \mathrm{M}$ be the Lie algebroid of $\mathcal{G}$ with anchor map $\nu: \mathrm{A} \rightarrow T \mathrm{M}$, and $\mathrm{E}$ be a vector bundle over M. Let $g_{\mathrm{A}}$ be a Riemannian metric on $\mathrm{A}$ and $\nabla^{\mathrm{E}}$ be an A-connection on $\mathrm{E}$ (for the definition of an A-connection and other details, see [3]). By right invariance, one obtains a (family of) Riemannian metric and connection, which we still denote by $\mathcal{G}_{\mathrm{A}}$ and $\nabla^{\mathrm{E}}$ respectively, on $\mathcal{G}_{x}$.

Definition 1.3. The Laplacian $\Delta^{\mathrm{E}}$ is the family of operators $\left\{\Delta_{x}^{\mathrm{E}}\right\}_{x \in \mathrm{M}}$, where

$$
\Delta_{x}^{\mathrm{E}}:=\sum_{i=1}^{n}\left(\nabla_{X_{i}}^{\mathrm{E}} \nabla_{X_{i}}^{\mathrm{E}}-\nabla_{\nabla_{X_{i}}^{\mathrm{E}} X_{i}}^{\mathrm{E}}\right)
$$


and $X_{i}$ is any local orthonormal basis of $T \mathcal{G}_{x}$.

Note that $\Delta^{\mathrm{E}}$ is elliptic, and its principal symbol does not depend on the chosen connection $\nabla^{\mathrm{E}}$.

In the shortest term, the heat kernel of $\Delta^{\mathrm{E}}$ is defined to be

Definition 1.4. The family $Q:=\left\{Q_{x}\right\}$, where for each $x \in \mathrm{M}, Q_{x}$ is the heat kernel of $\Delta_{x}^{\mathrm{E}}$, is called the heat kernel of $\Delta^{\mathrm{E}}$.

Equivalently, one can describe the operator $Q$ as a reduced kernel.

Definition 1.5. For any $\Psi=\left\{\Psi_{x}\right\}_{x \in \mathrm{M}} \in \Psi^{\infty}(\mathcal{G})$. The reduced kernel of $\Psi$ is defined to be the distribution

$$
K_{\Psi}(f):=\int_{\mathrm{M}} \mathbf{u}^{*}\left(\Psi\left(\mathbf{i}^{*} f\right)\right)(x) \mu_{\mathrm{M}}(x), \quad f \in C_{c}^{\infty}(\mathcal{G}),
$$

where $\mathbf{i}$ and $\mathbf{u}$ denote respectively the inversion and unit inclusion.

The important point here to note is that, while each $Q_{x}$ is defined by a smooth kernel (not compactly supported), $Q_{x}$ may not be a smooth family. In terms of reduced kernel, it is not clear that the reduced kernel of $Q$ is smooth, although it is easy to see that $Q$ is continuous.

Therefore, the regularity problem amounts to proving

Conjecture 1.6. Given certain groupoid $\mathcal{G} \rightrightarrows \mathrm{M}$ with $\mathrm{M}$ compact, the reduced heat kernel of $Q$ is smooth.

The main difficulty of establishing Conjecture 1.6 is that the groupoid $\mathcal{G}$ is in general non-compact, and there is no obvious transversal structure to enable one to compare different s-fibers.

1.2. Motivations and known results. The most notable partial result for Conjecture [1.6] is probably that of [4], where the author proves that the heat kernel on the holonomy groupoid is smooth. His proof uses heavily the constant rank of the (in general singular) foliation, which in turn enables one to naturally lift the orthogonal complement of the foliation on $\mathrm{M}$ to $\mathcal{G}$.

Not much is known about groupoids with non-constant rank. The case of manifolds with boundary was proven by Melrose [6], using explicit construction with boundary defining functions. Essentially the same argument was used by Albin [1, generalizing the result to edge calculus. However these techniques do not generalize easily to other groupoids, because there is no obvious definition of boundary function.

For groupoids not of the edge type, the only example we know is that of [9]. There, the author gives a criterion for the derivatives of the groupoid multiplication, and then verify that the symplectic groupoid of the Bruhat Poisson structure satisfies these properties. Our argument here will essentially follow similar lines.

Our main motivation of establishing the regularity is in line of the authors quoted above. For example, the renormalized integral considered in [1] and [9] requires the integrand to be differentiable. That in turn means it is necessary to establish Conjecture 1.6 in order to obtain a renormalized index formula by the heat kernel method. 
Another potentially interesting application of the results in this notes is on the polyhedral domain technique developed by Nistor et. al. The arguments developed in here may result in novel a-prior estimates for their numerical calculation.

Last but not the least, this notes can been seen as a sequel of [10, where the author attempts to generalize the classical theory of singular pseudo-differential calculus, using groupoids only. It appears the exponential coordinates defined here is central to many results (for example, smooth extension property) for boundary groupoids.

1.3. An overview of the argument. As far as we know, the exponential coordinates [7] is the only way to construct local complements of the s-foliation. In general, exponential coordinates are very arbitrary and therefore its use in estimating derivatives is limited. In the case of boundary groupoid with exponential isotropy subgroups (which implies these Lie groups are solvable and simply connected), the situation is easier as one only needs a finite cover of the singular invariant submanifolds.

In Section 2, we shall give a technical introduction of these exponential coordinates. Then we study the change in coordinates formulas, as well as groupoid multiplication under these coordinates.

In Section 3, we first recall the construction of the heat kernel by the method of Levi parametix. Then we directly show that on each exponential coordinate patch, derivatives of the Levi paramterix converges uniformly, thanks to the estimation done in Section 2. Hence we conclude that the groupoid heat kernel is smooth.

\section{Asymptotically EuClidean BOUNDARY GROUPOIDS}

Definition 2.1. Let $\mathcal{G} \rightrightarrows \mathrm{M}$ be a Lie groupoid with $\mathrm{M}$ compact. We say that $\mathcal{G}$ is a boundary groupoid if

(i) The anchor map $\nu: \mathrm{A} \rightarrow T \mathrm{M}$ stratifies $\mathrm{M}$ into invariant sub-manifolds $\mathrm{M}_{0}, \mathrm{M}_{1}, \cdots, \mathrm{M}_{r} \subset \mathrm{M}$

(ii) For all $k=0,1, \cdots r, \overline{\mathrm{M}}_{k}:=\mathrm{M}_{k} \bigcup \mathrm{M}_{k+1} \bigcup \cdots \cup \mathrm{M}_{r}$ are closed, immersed sub-manifolds of $\mathrm{M}$;

(iii) $\mathcal{G}_{0}:=\mathbf{s}^{-1}\left(\mathrm{M}_{0}\right)=\mathbf{t}^{-1}\left(\mathrm{M}_{0}\right) \cong \mathrm{M}_{0} \times \mathrm{M}_{0}$, the pair groupoid, and $\mathcal{G}_{k}:=$ $\mathbf{s}^{-1}\left(\mathrm{M}_{k}\right)=\mathbf{t}^{-1}\left(\mathrm{M}_{k}\right) \cong \mathrm{G}_{k} \times\left(\mathrm{M}_{k} \times \mathrm{M}_{k}\right)$ for some Lie groups $\mathrm{G}_{k}$;

(iv) For each $k$, there exists (unique) sub-bundles $\left.\overline{\mathrm{A}}_{k} \subset \mathrm{A}\right|_{\overline{\mathrm{M}}_{k}}$ such that $\left.\overline{\mathrm{A}}\right|_{\mathrm{M}_{k}}=$ $\operatorname{ker}\left(\left.\nu\right|_{\mathrm{M}_{k}}\right)$.

For simplicity, we shall also assume that $\mathrm{G}_{k}$ and $\mathrm{M}_{k}$ are connected, hence all s-fibers are connected.

On any boundary groupoids we have the following fundamental estimates [10]. Fix any Riemannian metric $\bar{g}$ on $\mathrm{M}$. For each $k \geq 1$, let $d\left(\cdot, \overline{\mathrm{M}}_{k}\right)$ be the distance function defined by $g$. For each $k \geq 0$, fix a function $\rho_{k} \in C^{\infty}(\mathrm{M})$ such that $\rho_{k}>0$ on $\mathrm{M} \backslash \overline{\mathrm{M}}_{k}$ and $\rho_{k}=d\left(\cdot, \overline{\mathrm{M}}_{k}\right)$ on some open set containing $\bar{M}_{k}$.

Lemma 2.2. For each $k$, there exists a constant $\omega_{k}$ such that for any $x$ lying in some open neighborhood of $\overline{\mathrm{M}}_{k}, X \in A_{x}$,

$$
\left|d \rho_{k} \circ \nu(X)\right| \leq \omega_{k} \rho_{k}(x)|X|_{g_{\mathrm{A}}} .
$$

Lemma 2.3. For each $k$, let $\omega_{k}$ be defined in the previous Lemma 2.2. Suppose further that $\left|d \rho_{k} \circ \nu(X)\right| \leq \omega_{k} \rho_{k}(x)|X|_{g_{\mathrm{A}}}$ for any $X \in \mathrm{A}$. Then for any $x \in \mathrm{M}, a, b \in$ 
$\mathcal{G}_{x}$,

$$
\omega_{k} d(a, b) \geq\left|\log \left(\frac{\rho_{k}(\mathbf{t}(b))}{\rho_{k}(\mathbf{t}(a))}\right)\right| .
$$

In this notes, we consider the simple case $\mathcal{G}=\left(\mathrm{M}_{0} \times \mathrm{M}_{0}\right) \bigsqcup\left(\mathrm{M}_{1} \times \mathrm{M}_{1} \times \mathbb{R}^{q}\right)$. In the following we write $\omega:=\omega_{1}, \rho:=\rho_{1}$, and we let $\operatorname{dim} \mathrm{M}_{1}=p, \operatorname{dim} \mathrm{M}_{0}=n=p+q$.

In the same vein, we recall the definitions of uniformly degenerate and nondegenerate in [10].

Definition 2.4. The groupoid $\mathcal{G}$ is said to be non-degenerate if there exist constants $\omega_{1}^{\prime}, \omega_{2}^{\prime}, \cdots, \omega_{r}^{\prime}>0$ such that

$$
|\nu(X)| \geq \omega_{k}^{\prime} \rho_{k}(x)|X|_{g_{\mathrm{A}}},
$$

for any $x \in \overline{\mathrm{M}}_{k-1}, X \in A_{x}$, and $X \perp \overline{\mathrm{A}}_{k}$; The groupoid $\mathcal{G}$ is said to be uniformly degenerate if there exist constants $\omega_{1}, \omega_{2}, \cdots, \omega_{r}, \omega_{1}^{\prime}, \cdots \omega_{r}^{\prime}>0$ and exponents $\lambda_{1}, \cdots \lambda_{r}$, $\lambda_{1}^{\prime}, \cdots, \lambda_{r}^{\prime} \geq 2$ such that

$$
\left|d \rho_{k} \circ \nu(X)\right| \leq \omega_{k}\left(\rho_{k}(x)\right)^{\lambda_{k}}|X|_{g_{\mathrm{A}}} \text { and }|\nu(X)| \geq \omega_{k}^{\prime}\left(\rho_{k}(x)\right)^{\lambda_{k}^{\prime}}|X|_{g_{\mathrm{A}}},
$$

for any $x \in \overline{\mathrm{M}}_{k-1}, X \in A_{x}$, and $X \perp \overline{\mathrm{A}}_{k}$.

2.1. The exponential map. Let $\mathcal{G} \rightrightarrows \mathrm{M}$ be a Lie groupoid with $\mathrm{M}$ compact. Denote the Lie algebroid of $\mathcal{G}$ by $\mathrm{A} \rightarrow \mathrm{M}$ and anchor map by $\nu$. Fix a Riemannian metric on A.

Given any smooth section $X \in \Gamma^{\infty}(\mathrm{A})$, denote by $X^{\mathbf{r}}$ the right invariant vector field on $\mathcal{G}$ with $\mathbf{s}^{*} X^{\mathbf{r}}=0$ and $\left.X^{\mathbf{r}}\right|_{\mathrm{M}}=X$. Since M is compact, it is standard that $X^{\mathbf{r}}$ is a complete vector field on $\mathcal{G}$, hence one has a well defined map

$$
\exp X: \mathrm{M} \rightarrow \mathcal{G}
$$

given by the flow of $X^{\mathbf{r}}$ form each $x \in \mathrm{M} \subset \mathcal{G}$. It is a well known fact that $\mathbf{t} \circ \exp X$ equals the flow of $\nu(X)$ on $\mathrm{M}$ and hence is a $\exp X$ is an admissible section. Define

$$
E_{X}:=d \mathbf{t} \circ d\left(\left.\exp X\right|_{\mathrm{A}}\right): \mathrm{A} \rightarrow \mathrm{A} .
$$

We list some basic properties of the exponential map [7], [5]:

(i) For any $X, Y \in C^{\infty}(\mathrm{A}), \exp X \exp Y=\exp Y \exp E_{X}$;

(ii) For any $x \in \mathrm{M},((\exp X)(x))^{-1}=\exp (-X)\left(E_{X}^{\nu}(x)\right)$, where $E_{X}^{\nu}: \mathrm{M} \rightarrow \mathrm{M}$ is the flow of $\nu(X)$.

Notation 2.5. For any collection of sections $Z_{I}=\left(Z_{1}, \cdots Z_{|I|}\right) \in \Gamma^{\infty}(\mathrm{A})$, denote

$$
\exp Z_{I}:=\exp Z_{|I|} \exp Z_{|I|-1} \cdots \exp Z_{2} \exp Z_{1} \text {. }
$$

For any $\mu=\left(\mu_{1}, \cdots, \mu_{|I|}\right) \in \mathbb{R}^{|I|}$, denote

$$
\exp \left(\mu \cdot Z_{I}\right):=\exp \mu_{|I|} Z_{|I|} \exp \mu_{|I|-1} Z_{|I|-1} \cdots \exp \mu_{2} Z_{2} \exp \mu_{1} Z_{1} .
$$

We specialize the construction of exponential coordinates in [7] to our case. Fix an orthonormal bases $\left\{Y_{1}, \cdots, Y_{q}\right\}$ of $\mathbb{R}^{q}$. We regard it as a basis of $T \mathrm{M}_{1} \times \mathbb{R}^{q}$ and extend to an orthonormal set of sections on some neighborhood of $\mathrm{M}_{1}$, which we still denote the extension by $\left\{Y_{1}, \cdots, Y_{q}\right\}$. Let $\tilde{V}_{\alpha} \subset \mathrm{M} \subset \mathcal{G}$ be a coordinate patch on $\mathrm{M}$, $\tilde{V}_{\alpha} \cap \mathrm{M}_{1} \neq \emptyset$. We may assume that $\left.\mathrm{A}\right|_{\tilde{V}_{\alpha}}$ is trivial. Fix a orthonormal basis

$$
\left\{Y_{1}, \cdots Y_{p}, X_{1}^{(\alpha)}, \cdots, X_{q}^{(\alpha)}\right\} \subset \Gamma^{\infty}\left(\left.\mathrm{A}\right|_{\tilde{V}_{\alpha}}\right),
$$


and extend $X^{(\alpha)}$ to smooth compactly supported sections.

Notation 2.6. For any $\tau \in \mathbb{R}^{q}$, denote by

$$
\|\tau\|:=\left|\tau_{1}\right|+\cdots+\left|\tau_{q}\right|,
$$

the 1-norm of $\tau$. Given $r, M>0$, define

$$
T(r, M):=\left\{(x, \tau) \in \mathrm{M} \times \mathbb{R}^{q}: \rho(x)<r e^{-M\|\tau\|}\right\} .
$$

Clearly $T\left(r^{\prime}, M^{\prime}\right) \subseteq T(r, M)$ whenever $r^{\prime} \geq r, M^{\prime} \geq M$.

Definition 2.7. Given $\left\{Y_{1}, \cdots Y_{p}, X_{1}^{(\alpha)}, \cdots, X_{q}^{(\alpha)}\right\}$ as above, and a arbitrary collection $Z_{I}=\left\{Z_{1}, \cdots, Z_{|I|}\right\} \subset \operatorname{Span}_{\mathbb{R}}\left\{X_{1}^{(\alpha)}, \cdots, X_{q}^{(\alpha)}\right\}$, (over all $\alpha$ ) define the function $\mathbf{x}_{Z_{I}}^{(\alpha)}: V_{\alpha} \times \mathbb{R}^{p+q} \rightarrow \mathcal{G}$,

$$
\mathbf{x}_{Z_{I}}^{(\alpha)}(x, \mu, \tau):=\exp \tau \cdot Y \exp \mu \cdot X^{(\alpha)} \exp Z_{I}(x) .
$$

Lemma 2.8. There exists $\varepsilon, r_{0}, M>0$ such that $\mathbf{x}^{(\alpha)}$ is a local diffeomorphism from $(-\varepsilon, \varepsilon)^{p} \times T\left(r_{0}, M\right)$ onto its image.

Proof. By the inverse function theorem it suffices to prove that the differential of $\mathbf{x}$ is invertible. It is straightforward to compute for any $a=\mathbf{x}(x, \mu, \tau)$, the 'coordinate vector fields' are given by

$$
\begin{aligned}
\partial_{\tau_{i}}(a) & =d \mathbf{r}_{a}\left(E_{\tau_{q} Y_{q}} \cdots E_{\tau_{i+1} Y_{i+1}} Y_{i}\left(E_{\tau_{i} Y_{i}}^{\nu} \cdots E_{\tau_{1} Y_{1}}^{\nu} E_{\mu \cdot X^{(\alpha)}}^{\nu} E_{Z_{I}}^{\nu}(x)\right)\right), \\
\partial_{\mu_{i^{\prime}}}(a) & =d \mathbf{r}_{a}\left(E_{\tau \cdot Y} E_{\mu_{p} X_{p}^{(\alpha)}}^{(\alpha)} \cdots E_{\mu_{i^{\prime}+1} X_{i^{\prime}+1}^{(\alpha)}} Y_{i^{\prime}}\left(E_{\mu_{i^{\prime}} X_{i^{\prime}}^{(\alpha)}}^{\nu} \cdots E_{\mu_{1} X_{1}^{(\alpha)}}^{\nu} E_{Z_{I}}^{\nu}(x)\right)\right) .
\end{aligned}
$$

In view that $d \mathbf{s}\left(\partial_{x}\right)=\partial_{x}$, it suffices to show that $\partial_{\tau_{i}}, \partial_{\mu_{i^{\prime}}}$ is a basis of $T^{\perp} \mathcal{G}$. Following Equation (3), one writes

$$
\begin{gathered}
E_{\tau_{q} Y_{q}} \cdots E_{\tau_{i+1} Y_{i+1}} Y_{i}\left(E_{\tau_{i} Y_{i}}^{\nu} \cdots E_{\tau_{1} Y_{1}}^{\nu} E_{\mu \cdot X^{(\alpha)}}^{\nu} E_{Z_{I}^{\alpha}}^{\nu}(x)\right) \\
\left.:=\left(\sum_{j=1}^{q} w_{j i} Y_{j}+\sum_{j^{\prime}=1}^{p} w_{j^{\prime} i} X_{j^{\prime}}^{(\alpha)}\right)\left(E_{\tau \cdot Y}^{\nu} E_{\mu \cdot X^{(\alpha)}}^{\nu} E_{Z_{I}^{\alpha}}^{\nu}\right)(x)\right) \\
E_{\tau . Y} E_{\mu_{p} X_{p}^{(\alpha)}} \cdots E_{\mu_{i^{\prime}+1} X_{i^{\prime}+1}^{(\alpha)}} X_{i^{\prime}}^{(\alpha)}\left(E_{\mu_{i^{\prime}} \cdot X_{i^{\prime}}^{(\alpha)}}^{\nu} \cdots E_{\mu_{1} X_{1}^{(\alpha)}}^{\nu} E_{Z_{I}^{\alpha}}^{\nu}(x)\right) \\
\left.:=\left(\sum_{j=1}^{q} w_{j i^{\prime}} Y_{j}+\sum_{j^{\prime}=1}^{p} w_{j^{\prime} i^{\prime}} X_{j^{\prime}}^{(\alpha)}\right)\left(E_{\tau \cdot Y}^{\nu} E_{\mu \cdot X^{(\alpha)}}^{\nu} E_{Z_{I}^{\alpha}}^{\nu}\right)(x)\right) .
\end{gathered}
$$

Observe that at $x \in \mathrm{M}_{1}, w_{j i}=1$ if $i=j, w_{j^{\prime} i^{\prime}}=1$, and zero otherwise. Moreover, it is clear that the $x$-derivatives of $w$ are bounded by $C_{1} e^{M_{1}\|\tau\|}$. It follows that for any $(x, \tau) \in T\left(r, M_{1}\right)$ with $r>0$ sufficiently small, we can write

$$
\left(\begin{array}{cc}
w_{j i} & w_{j^{\prime} i} \\
w_{j i^{\prime}} & w_{j^{\prime} i^{\prime}}
\end{array}\right)=I+\left(\begin{array}{cc}
w_{j i}^{\prime} & w_{j^{\prime} i}^{\prime} \\
w_{j i^{\prime}}^{\prime} & w_{j^{\prime} i^{\prime}}^{\prime}
\end{array}\right)
$$

with the last term on the right hand side uniformly small, hence $\left(\begin{array}{cc}w_{j i} & w_{j^{\prime} i} \\ w_{j i^{\prime}} & w_{j^{\prime} i^{\prime}}\end{array}\right)$ is invertible, and the assertion follows.

In the rest of this notes we shall make the following additional assumption: 
Assumption 2.9. For some $T\left(r_{0}, M\right)$, the map $\mathbf{x}^{(\alpha)}$ are diffeomorphisms. In other words, $\mathbf{x}^{(\alpha)}$ defines a local coordinate patch.

Notation 2.10. We denote the image of $\mathbf{x}^{(\alpha)}$ by $U_{\alpha}$. For fixed $\mu, \tau$, denote $\mathbf{x}^{(\alpha)}(\mu, \tau)$ to be the (local) section $\exp \tau \cdot Y \exp \mu \cdot X^{(\alpha)} \exp Z_{I_{\alpha}}$.

Remark 2.11. In general, if $\mathrm{G}$ is not Abelian, then $\left|E_{\tau \cdot Y}\right|$ is not uniformly bounded. If $\mathrm{G}$ is nilpotent, then $\left|E_{\tau \cdot Y}\right|$ is bounded by estimate of the form $e^{(\log |\tau|)^{2}}$. See [10, Appendix B].

2.2. Groupoid multiplication. The main advantage of the exponential coordinates we constructed, is that the groupoid multiplication can be described rather explicitly.

To begin with, consider $\exp \tau_{i} Y_{i} \mathbf{x}^{(\alpha)}(x, \mu, \tau)$. Recall that by definition, $\exp t Y_{i}$ $\mathbf{x}^{(\alpha)}(x, \mu, \tau)$ is just the integral curve of the vector field $Y_{i}^{\mathbf{r}}$ from $\mathbf{x}^{(\alpha)}(x, \mu, \tau)$, evaluated at $\tau_{i}$. So we write

$$
Y_{i}^{\mathbf{r}}\left(\mathbf{x}^{(\alpha)}(x, \mu, \tau)\right):=\sum_{j=1}^{q} v_{j i}(x, \mu, \tau) \partial_{\tau_{j}}(x, \mu, \tau)+\sum_{j^{\prime}=1}^{p} v_{j^{\prime} i}(x, \mu, \tau) \partial_{\mu_{j^{\prime}}}(x, \mu, \tau) .
$$

In other words,

$$
\begin{array}{r}
Y_{i}\left(E_{\tau^{(\alpha)} \cdot Y}^{\nu} E_{\mu^{(\alpha)} \cdot X^{(\alpha)}}^{\nu} E_{X_{I}}^{\nu}(x)\right)=\sum_{j=1}^{q} v_{j i} E_{\tau_{q} Y_{q}} \cdots E_{\tau_{j+1} Y_{j+1}} Y_{j}\left(E_{\tau_{j} Y_{j}}^{\nu} \cdots E_{\tau_{1} Y_{1}}^{\nu} E_{\mu \cdot X^{(\alpha)}}^{\nu}(x)\right) \\
+\sum_{j^{\prime}=1}^{p} v_{j^{\prime} i} E_{\tau \cdot Y} E_{\mu_{p} X_{p}^{(\alpha)}} \cdots E_{\mu_{j^{\prime}+1} X_{j^{\prime}+1}^{(\alpha)}} X_{j^{\prime}}^{(\alpha)}\left(E_{\mu_{j^{\prime}} X_{j^{\prime}}^{(\alpha)}}^{\nu} \cdots E_{\mu_{1}^{(\alpha)} X_{1}^{(\alpha)}}^{\nu}(x)\right) .
\end{array}
$$

Note that $\left(\begin{array}{cc}v_{j i} & v_{j^{\prime} i} \\ v_{j i^{\prime}} & v_{j^{\prime} i^{\prime}}\end{array}\right)=\left(\begin{array}{cc}w_{j i} & w_{j^{\prime} i} \\ w_{j i^{\prime}} & w_{j^{\prime} i^{\prime}}\end{array}\right)^{-1}$, with the right hand side defined in the proof of Lemma 2.8 .

It is now straightforward to write

$$
\exp t Y_{i} \mathbf{x}^{(\alpha)}(x, \mu, \tau):=\mathbf{x}^{(\alpha)}(x, \varphi(x, \mu, \tau, t), \psi(x, \mu, \tau, t)),
$$

where $(\varphi(x, \mu, \tau, t), \psi(x, \mu, \tau, t))$ is just the solution of the system of ODE

$$
\begin{aligned}
& \frac{d \varphi_{j^{\prime}}(x, \mu, \tau, t)}{d t}=v_{j^{\prime} i}(x, \varphi(x, \mu, \tau, t), \psi(x, \mu, \tau, t)) \\
& \frac{d \psi_{j}(x, \mu, \tau, t)}{d t}=v_{j i}(x, \varphi(x, \mu, \tau, t), \psi(x, \mu, \tau, t))
\end{aligned}
$$

with the obvious initial condition $\varphi(x, \mu, \tau, 0)=\mu, \psi(x, \mu, \tau, 0)=\tau$.

As in Equation (4), observe that when $x \in \mathrm{M}_{1}, \partial_{\tau_{i}}=Y_{i}^{\mathbf{r}}$. In other words $v_{j i}=\delta_{j i}$, and $v_{j^{\prime} i}=0$. Clearly, the derivatives $\partial_{x} v_{j i}(x, \mu, \tau), \partial_{x} v_{j^{\prime} i}(x, \mu, \tau)$ are bounded by $C_{2} e^{M_{2}\|\tau\|}$. It follows that we have the a-prior estimate

$$
\left|\psi_{j}(x, \mu, \tau, t)-\tau_{i}\right| \leq \rho(x) C_{2} e^{M_{2}\|\tau\|_{1}} \leq C_{2} r t,
$$

whenever $x \in T(r, K), K \geq M_{2}$. In particular, if $(x, \tau) \in T\left(r e^{-q C_{2} r t_{0}}, K\right)$, then $\psi(x, \mu, \tau, t)$ is well defined for $-t_{0} \leq t \leq t_{0}$ since $(x, \psi(x, \mu, \tau, t)) \in T(r, K)$. 
In turn, the a-prior estimate implies

$$
\begin{array}{r}
\left|v_{j i}(x, \varphi(x, \mu, \tau, t), \psi(x, \mu, \tau, t))-\delta_{j i}\right| \leq \rho(x) C_{2} e^{M_{2}\left(\|\tau\|+q C_{2} r t\right)} \\
\left|v_{j^{\prime} i}(x, \varphi(x, \mu, \tau, t), \psi(x, \mu, \tau, t))\right| \leq \rho(x) C_{2} e^{M_{2}\left(\|\tau\|+q C_{2} r t\right)} .
\end{array}
$$

Integrating with respect to $t$, we get

$$
\begin{aligned}
\left|\psi_{j}(x, \mu, \tau, t)-\delta_{j i} t-\tau_{j}\right| & \leq \rho(x)(q r)^{-1} e^{M_{2}\left(\|\tau\|+q C_{2} r t\right)} \\
\left|\varphi_{j^{\prime}}(x, \mu, \tau, t)-\mu_{j^{\prime}}\right| & \leq \rho(x)(q r)^{-1} e^{M_{2}\left(\|\tau\|+q C_{2} r t\right)} .
\end{aligned}
$$

The case for $\exp t Z \mathbf{x}^{(\alpha)}(x, \mu, \tau), t \in[0,1]$, where $Z \in \operatorname{Span}\left\{X^{(\alpha)}\right\}$ (over all $\alpha$ ) is similar. Again one writes

$$
\exp t Z \mathbf{x}^{(\alpha)}(x, \mu, \tau)=\mathbf{x}^{(\alpha)}(x, \tilde{\varphi}(x, \mu, \tau, t), \tilde{\psi}(x, \mu, \tau, t)),
$$

and one has

$$
\begin{aligned}
& \frac{d \tilde{\varphi}_{j^{\prime}}(x, \mu, \tau, t)}{d t}=v_{j^{\prime} i^{\prime}}(x, \tilde{\varphi}(x, \mu, \tau, t), \tilde{\psi}(x, \mu, \tau, t)) \\
& \frac{d \tilde{\psi}_{j}(x, \mu, \tau, t)}{d t}=v_{j i^{\prime}}(x, \tilde{\varphi}(x, \mu, \tau, t), \tilde{\psi}(x, \mu, \tau, t)) .
\end{aligned}
$$

Using the same arguments as above, we get $w_{j i^{\prime}}(x, \tilde{\varphi}, \tilde{\psi})=0$ for any $x \in \mathrm{M}_{1}$ and

$$
\left|\tilde{\psi}_{j}(x, \mu, \tau, t)-\tau_{j}\right| \leq \rho(x)(q r)^{-1} e^{M_{2}\left(\|\tau\|+q C_{2} r t\right)} .
$$

Remark 2.12. Observe that one can also write

$$
\exp t Z \mathbf{x}^{(\alpha)}(x, \mu, \tau)=\exp _{\tau \cdot Y} \exp _{E_{\tau \cdot Y} t Z} \mathbf{x}^{(\alpha)}(x, \mu, 0)(x) .
$$

The techniques leading to Equation (6) can be further refined to give an estimate of the derivatives of the multiplication map. First consider $d\left(\exp t Y_{i}\right) \partial_{x}\left(\mathbf{x}^{(\alpha)}(x, \tau, \mu)\right)$. Clearly, one has

$$
d\left(\exp t Y_{i}\right) \partial_{x}\left(\mathbf{x}^{(\alpha)}(x, \tau, \mu)\right)=\left(\partial_{x}+\sum_{j=1}^{q}\left(\partial_{x} \psi_{j}\right) \partial_{\tau_{j}}+\sum_{j^{\prime}=1}^{p}\left(\partial_{x} \varphi_{j^{\prime}}\right) \partial_{\mu_{j}}\right)\left(\mathbf{x}^{(\alpha)}(x, \varphi, \psi)\right) .
$$

To estimate $\partial_{x} \psi(x, \mu, \tau, t)$ and $\partial_{x} \varphi(x, \mu, \tau, t)$, differentiate Equation (5) with respect to $x$ to get

$$
\begin{array}{r}
\frac{d}{d t} \partial_{x} \varphi_{j^{\prime}}(x, \mu, \tau, t) \\
=\left(\frac{\partial v_{j^{\prime} i}}{\partial x}+\sum_{i^{\prime}=k}^{p} \frac{\partial \varphi_{k^{\prime}}}{\partial x} \frac{\partial v_{j^{\prime} i}}{\partial \mu_{k^{\prime}}}+\sum_{i=k}^{q} \frac{\partial \psi_{k}}{\partial x} \frac{\partial v_{j^{\prime} i}}{\partial \tau_{k}}\right)(x, \varphi(x, \mu, \tau, t), \psi(x, \mu, \tau, t)) \\
\frac{d}{d t} \partial_{x} \psi_{j}(x, \mu, \tau, t) \\
=\left(\frac{\partial v_{j i}}{\partial x}+\sum_{k^{\prime}=1}^{p} \frac{\partial \varphi_{k^{\prime}}}{\partial x} \frac{\partial v_{j i}}{\partial \mu_{k^{\prime}}}+\sum_{k=1}^{q} \frac{\partial \psi_{k}}{\partial x} \frac{\partial v_{j i}}{\partial \tau_{k}}\right)(x, \varphi(x, \mu, \tau, t), \psi(x, \mu, \tau, t)) .
\end{array}
$$

Moreover, $\partial_{x} \varphi(x, \mu, \tau, 0)=\partial_{x} \psi(x, \mu, \tau, 0)=0$.

Recall that $\left|\partial_{x} v_{j_{1}}(x, \varphi(x, \mu, \tau, t), \psi(x, \mu, \tau, t))\right| \leq \rho(x)(q r)^{-1} e^{M_{2}\left(\|\tau\|+q C_{2} r|t|\right)}$, for some constants $C_{2}, M_{2}>0$. As for the terms in the summation, note that at $x \in \mathrm{M}_{1}, v_{j 1}$ are just constants, therefore $\partial_{\tau_{i}} v_{j 1}, \partial_{\mu_{i^{\prime}}} v_{j 1}$ vanish. It follows that $\partial_{\tau_{i}} v_{j 1}, \partial_{\mu_{i^{\prime}}} v_{j 1}$ are 
bounded for $(x, \tau)$ lying in some $T\left(r_{0}, K\right)$. In other words, we have a linear equation of the form

$$
\frac{d}{d t}\left(\begin{array}{c}
\partial_{x} \psi \\
\partial_{x} \varphi
\end{array}\right)(x, \mu, \tau, t) \leq A\left(\begin{array}{c}
\partial_{x} \psi \\
\partial_{x} \varphi
\end{array}\right)(x, \mu, \tau, t)+\rho(x)(q r)^{-1} e^{M_{2}\left(\|\tau\|+q C_{2} r t\right)} .
$$

for some constant matrix $A$. Integrating, one obtains:

Lemma 2.13. One has

$$
\left(d \exp t Y_{i}\right) \partial_{x}\left(\mathbf{x}^{(\alpha)}(x, \tau, \mu)\right)=\partial_{x}+X
$$

where $X \in T^{\perp} \mathcal{G}$ and satisfies an estimate the form

$$
|X| \leq C_{3} e^{M_{3}(\|\tau\|+|t|)},
$$

for some constants $C_{3}, M_{3}$.

The case for $\partial_{\tau_{k}}$ and $\partial_{\mu_{k^{\prime}}}$ is much simpler. Observe that both vector lies in $T^{\perp} \mathcal{G}$, therefore

$$
\left(d \exp t Y_{i}\right) \partial_{\tau_{k}}\left(\mathbf{x}^{(\alpha)}(x, \tau, \mu)\right)=d \mathbf{r}_{\exp _{t Y_{i}} \mathbf{x}^{(\alpha)}(x, \tau, \mu)}\left(E_{t Y_{i}}\left(d \mathbf{r}_{\mathbf{x}^{(\alpha)}(x, \tau, \mu)}^{-1} \partial_{\tau_{k}}\right)\right) .
$$

Since the metric $g$ on $T^{\perp} \mathcal{G}$ is right invariant, we have

$$
\left|\left(d \exp t Y_{i}\right) \partial_{\tau_{k}}\right| \leq C_{4} e^{M_{4} t}\left|\partial_{\tau_{k}}\right|
$$

for some constants $C_{4}, M_{4}$, and clearly similar estimate holds for $\partial_{\mu_{k^{\prime}}}$. Here, the important point to note is that the estimate does not depend on $(x, \mu, \tau)$.

2.3. Change of coordinates. In this section we turn to the change of coordinate formulas. Observe that suppose one has $\mathbf{x}^{(\beta)}(x, \mu, \tau)=\mathbf{x}^{(\alpha)}(x, \varphi(x, \mu, \tau), \psi(x, \mu, \tau))$, then

$$
\mathbf{x}^{(\beta)}(x, \mu, \tau)=\exp \tau \cdot Y \exp \mathbf{x}^{(\beta)}(x, \mu, 0)=\exp \tau . Y \mathbf{x}^{(\alpha)}(x, \varphi(x, \mu, 0), \psi(x, \mu, 0)) .
$$

Suppose that $(\varphi(x, \mu, 0)-2 \varepsilon, \varphi(x, \mu, 0)+2 \varepsilon)^{p} \subset\left(-\varepsilon_{\alpha}, \varepsilon_{\alpha}\right)^{p}$, where

$$
\mathbf{x}^{(\alpha)}:\left(-\varepsilon_{\alpha}, \varepsilon_{\alpha}\right)^{p} \times\left(V_{\alpha} \bigcap T(r, K)\right) \rightarrow \mathcal{G} .
$$

Then, by possibly by restricting $T(r, K), \varphi(x, \mu, \tau)$ and $\psi(x, \mu, \tau)$ can be computed using Equation (6). In particular, the second equation of (6) implies $\varphi(x, \mu, \tau)$ still lies in the domain of $\mathbf{x}^{(\alpha)}$. Moreover, repeated use of the first equation of (6) now implies that

$$
\begin{gathered}
\left\|\psi\left(x, \mu, \tau_{1}, 0, \cdots, 0\right)-\psi(x, \mu, 0, \cdots, 0)-\tau\right\| \leq \rho(x) C_{2} e^{M_{2}\left(\left|\tau_{1}\right|+\|\psi(x, \mu, 0, \cdots, 0)\|\right)} \\
\left\|\psi\left(x, \mu, \tau_{1}, \tau_{2}, 0, \cdots, 0\right)-\psi\left(x, \mu, \tau_{1}, 0, \cdots, 0\right)-\tau\right\| \leq \rho(x) C_{2} e^{M_{2}\left(\left|\tau_{2}\right|+\left\|\psi\left(x, \mu, \tau_{1}, 0, \cdots, 0\right)\right\|\right)} \\
\leq \rho(x) C_{2} e^{M_{2}\left(\left|\tau_{2}\right|+\left|\tau_{1}\right|+\rho(x) C_{2} e^{M_{2}\left(\left|\tau_{1}\right|+\|\psi(x, \mu, 0)\|\right)}\right)} \\
\vdots \\
\|\psi(x, \mu, \tau)-\tau\| \leq \rho(x) C_{4} e^{M_{4}\|\tau\|}
\end{gathered}
$$

Here, note the assumption $(x, \tau) \in T(r, K), K \geq C_{2}$, implies all term on the right hand side are bounded. On the other hand, Lemma 2.13 implies that one has estimations for the change of coordinate vector fields:

$$
\left\|\partial_{x} \psi(x, \mu, \tau)\right\| \leq C_{5} e^{M_{5}\|\tau\|} .
$$


Fix a collection of coordinate charts

$$
\mathbf{x}^{(\alpha)}:\left(-\varepsilon_{\alpha}, \varepsilon_{\alpha}\right)^{p} \times\left(V_{\alpha} \bigcap T(r, C)\right) \rightarrow \mathcal{G}
$$

and $\varepsilon>0$ such that for all $x, \in V_{\alpha}, \mu^{(\alpha)} \in\left(-\varepsilon^{(\alpha)}, \varepsilon^{(\alpha)}\right)$, there exists $\beta$ (possibly same as $\alpha$ ) such that

$$
\mathbf{x}^{(\alpha)}\left(x, \mu^{(\alpha)}, 0\right)=\mathbf{x}^{(\beta)}\left(x, \mu^{(\beta)}, \tau^{(\beta)}\right)
$$

for some $\mu^{(\beta)} \in \mathbb{R}^{p}$ satisfying $\left(-2 \varepsilon+\mu^{(\beta)}, 2 \varepsilon+\mu^{(\beta)}\right) \subseteq\left(-\varepsilon_{\beta}, \varepsilon_{\beta}\right)^{p}$.

Since the product

$$
\mathbf{x}^{\left(\alpha_{2}\right)}\left(E_{\mathbf{x}^{\left(\alpha_{1}\right)}\left(\mu_{1}, \tau_{1}\right)}^{\nu}(x), \mu_{2}, \tau_{2}\right) \mathbf{x}^{\left(\alpha_{1}\right)}\left(x, \mu_{1}, \tau_{1}\right)
$$

is by definition just an iteration of left multiplication by admissible sections of the form $\exp Z$ then followed by $\exp \tau_{2} \cdot Y$. In particular, we may change coordinates so that

$$
\mathbf{x}^{\left(\alpha_{1}\right)}\left(x, \mu_{1}, \tau_{1}\right)=\mathbf{x}^{\left(\beta_{1}\right)}\left(x, \mu_{1}^{\prime}, \tau_{1}^{\prime}\right),
$$

where $\left(-\varepsilon+\mu^{\prime}, \varepsilon+\mu^{\prime}\right) \subseteq\left(-\varepsilon_{\beta}, \varepsilon_{\beta}\right)^{p}$. Then by Equation (6) we have

$$
\exp Z_{1} \mathbf{x}^{\left(\beta_{1}\right)}\left(x, \mu_{1}^{\prime}, \tau_{1}^{\prime}\right)=\mathbf{x}^{\left(\beta_{1}\right)}\left(x, \mu_{1}^{\prime \prime}, \tau_{1}^{\prime \prime}\right)
$$

such that $\left\|\tau_{1}^{\prime \prime}-\tau_{1}\right\| \leq \rho(x) C_{6} e^{M_{6}\left\|\tau_{1}\right\|}$ for some $C_{6}, M_{6} \geq 0$. Obviously the same arguments can be iterated to compute (11).

At the same time, one can estimate of the differential of each successive left multiplication by Lemma 2.13 and Equation (10). Hence we conclude that

Theorem 2.14. There exist some $r, H>0$ such that whenever $x \in B\left(r e^{-k H}\right),\left\|\tau_{i}\right\| \leq$ $1, i=1,2, \cdots k$,

$$
\mathbf{x}^{\left(\alpha_{k}\right)}\left(\mu_{k}, \tau_{k}\right) \cdots \mathbf{x}^{\left(\alpha_{1}\right)}\left(\mu_{1}, \tau_{1}\right)(x)=\mathbf{x}^{(\beta)}(x, \mu, \tau)
$$

for some exponential coordinate chart $\mathbf{x}^{(\beta)}$. Moreover, one has

$$
d\left(\mathbf{x}^{\left(\alpha_{k}\right)}\left(\mu_{k}, \tau_{k}\right) \cdots \mathbf{x}^{\left(\alpha_{1}\right)}\left(\mu_{1}, \tau_{1}\right)\right)\left(\partial_{x}\right)=\partial_{x}+V,
$$

where $\partial_{x}$ on the right hand side is the coordinate vector field on $\mathbf{x}^{(\beta)}$, and $V \in T^{\perp} \mathcal{G}$ (i.e. $V$ is spanned by $\partial_{\tau_{j}^{(\beta)}}$ and $\partial_{\mu_{j^{\prime}}^{(\beta)}}$ ), is such that

$$
|V| \leq C_{7} e^{M_{7} k}
$$

for some constant $C_{7}, M_{7}>0$.

\section{THE GROUPOID HEAT KERNEL}

In terms of reduced kernel and convolution product, the heat kernel is defined as:

Definition 3.1. A (groupoid) Heat kernel of $\Delta$ is a continuous section

$$
Q \in \Gamma^{0}(\mathcal{G} \times(0, \infty)),
$$

such that $Q(a, t), Q\left(a^{-1}, t\right)$ are smooth when restricted to all $\mathcal{G}_{x} \times(0, \infty)$, and satisfies:

(i) The heat equation

$$
\left(\partial_{t}+\Delta\right) Q(a, t)=0
$$


(ii) The initial condition

$$
\lim _{t \rightarrow 0^{+}} Q \circ u=u, \quad \forall u \in \Gamma_{c}^{\infty}(\mathcal{G}),
$$

where $\circ$ denotes the convolution product

$$
\kappa_{0} \circ \kappa_{1}(a):=\int_{b \in \mathcal{G}_{(}(a)} \kappa_{0}\left(a b^{-1}\right) \kappa_{1}(b) d b, \quad \forall \kappa_{1}, \kappa_{2} \in C_{c}^{0}(\mathcal{G}), a \in \mathcal{G} .
$$

3.1. Construction of the heat kernel. In this section, we briefly review how one constructs the heat kernel of a generalized Laplacian operator [2]. For simplicity, we only consider the scalar case.

By [2], there exists a formal power series $\Phi(a, t):=\sum_{i=1}^{\infty} t^{i} \Phi_{i}(a)$ that satisfies the equation

$$
\left(\partial_{t}+\Delta\right)(4 \pi t)^{-\frac{n}{2}} e^{-\frac{d(a, \mathbf{s}(a))^{2}}{4 t}} \Phi(a, t)=0 .
$$

Fix a cutoff function $\phi$ supported on $B\left(\mathrm{M}, \varrho_{0}\right)$ such that $\phi=1$ on the smaller set $B\left(\mathrm{M}, \frac{\varrho_{0}}{2}\right):=\left\{a \in \mathcal{G}: d(a, \mathbf{s}(a)) \leq \frac{\varrho_{0}}{2}\right\}$. Write

$$
G_{N}(a, t):=\phi(a)(4 \pi t)^{-\frac{n}{2}} e^{-\frac{d(a, \mathbf{s}(a))^{2}}{4 t}} \sum_{i=1}^{N} t^{i} \Phi_{i}(a), \quad t \in(0, \infty) .
$$

For each $N>\frac{n}{2}$, define the sections $R_{n}^{(k)} \in \Gamma^{\infty}(\mathcal{G} \times[0, \infty))$ :

$$
\begin{aligned}
R_{N}^{(1)} & :=\left(\partial_{t}+\Delta\right) G_{N} \\
R_{N}^{(k)} & :=\int_{0}^{t} R_{N}(\cdot, t-s) \circ R_{N}^{(k-1)}(\cdot, s) d s \\
& =\int_{0}^{t} \int_{\mathbf{s}^{-1}(a)} R_{n}\left(a b^{-1}, t-s\right) R_{N}^{(k-1)}(b, \tau) \mu_{\mathbf{s}(a)}(b) d s \\
Q_{N}^{(0)} & :=G_{N} \\
Q_{N}^{(k)} & :=\int_{0}^{t} G_{N}(\cdot, t-s) \circ R_{N}^{(k)}(\cdot, s) d s, \quad k \geq 1 \\
& =\int_{s \in \Sigma_{k}} G_{N}(\cdot, t-\|s\|) \circ R_{N}^{(1)}\left(\cdot, s_{1}\right) \circ \cdots \circ R_{N}^{(1)}\left(\cdot, s_{k}\right) d s,
\end{aligned}
$$

where $\Sigma_{k}:=\left\{\left(s_{1}, \cdots, s_{k}\right) \in \mathbb{R}^{k}, s_{1}, \cdots, s_{k} \geq 0, s_{1}+\cdots+s_{k} \leq t\right\}$, and $d s$ is the Lebesgue measure. Then the heat kernel is constructed by taking the $\operatorname{sum} Q:=$ $\sum_{k=0}^{\infty}(-1)^{k} Q_{N}^{(k)}(\cdot, t)$.

To simplify notation, we shall write $R_{N}:=R^{(1)}$, and omit the reference to $t$.

3.2. $C^{l}$ estimation near $\mathcal{G}_{1}$. Let $\left\{\mathbf{x}^{(\alpha)}\right\}$ be the family coordinate patches defined in Section 2.3. Define $U_{\alpha}^{0}:=\left\{\mathbf{x}^{(\alpha)}(x, \mu, \tau): \tau \in(-1,1)^{q}\right\}$ and $U^{0}:=\bigcup_{\alpha} U_{\alpha}^{0}$. Fix a partition of unity $\theta_{\alpha}$ of $U$ subordinated to $U_{\alpha}$.

In the construction of $G_{N}$, we may assume that $\varrho$ is sufficiently small, such that

$$
\left(\mathbf{s}^{*} \rho\right)^{-1}\left[0, \frac{r}{2}\right) \bigcap B(\mathrm{M}, \varrho) \subset U^{0} .
$$

Fix another cutoff function $\chi \in C_{c}^{\infty}(\mathbb{R})$ such that $\chi=1$ on $\left[0, \frac{1}{2}\right]$ and 0 on $[1, \infty)$. 
Given smooth compactly supported functions $\kappa_{0}, \cdots, \kappa_{k}$ on $\mathcal{G}$, it is straightforward to write for any $a \in \mathcal{G}$ :

$$
\begin{aligned}
& \kappa_{0} \circ \cdots \circ \kappa_{k}(a) \\
& \quad=\int_{b_{1} \in \mathcal{G}_{\mathbf{s}(a)}} \int_{b_{2} \in \mathcal{G}_{\mathbf{t}\left(b_{1}\right)}} \cdots \int_{b_{k} \in \mathcal{G}_{\mathbf{t}\left(b_{k-1}\right)}} \kappa_{0}\left(a b_{1}^{-1} \cdots b_{k}^{-1}\right) \kappa_{1}\left(b_{1}\right) \cdots \kappa_{k}\left(b_{k}\right) d b_{k} \cdots d b_{1} .
\end{aligned}
$$

Suppose that $\kappa_{i}$ is supported on $U^{0}$ for all $i$. Then one can write

$$
\begin{array}{r}
\left(\kappa_{0} \circ \cdots \kappa_{k}\right)(a)=\sum_{\alpha_{1}, \cdots, \alpha_{k}} \int_{\left(\mu_{i}, \tau_{i}\right) \in(-1,1)^{k(p+q)}} \kappa_{0}\left(a\left(\mathbf{x}^{\left(\alpha_{k}\right)}\left(\mu_{k}, \tau_{k}\right) \cdots \mathbf{x}^{\left(\alpha_{1}\right)}\left(\mu_{1}, \tau_{1}\right)(x)\right)^{-1}\right) \\
\times \prod_{i=1}^{k} \theta_{\alpha_{i}} \kappa_{i}\left(\mathbf{x}^{\left(\alpha_{i}\right)}\left(E_{\mathbf{x}^{\left(\alpha_{i-1}\right)}\left(\mu_{i-1}, \tau_{i-1}\right)}^{\nu} \cdots E_{\mathbf{x}^{\left(\alpha_{1}\right)}\left(\mu_{1}, \tau_{1}\right)}^{\nu}(x), \mu_{i}, \tau_{i}\right)\right) \prod_{i=1}^{k} d \mu_{i} d \tau_{i} .
\end{array}
$$

Consider differentiating the integrand with respect to $x$.

It is clear that $\left(d E_{\mathbf{x}^{\left(\alpha_{i-1}\right)}\left(\mu_{i-1}, \tau_{i-1}\right)}^{\nu} \cdots E_{\mathbf{x}^{\left(\alpha_{1}\right)}\left(\mu_{1}, \tau_{1}\right)}^{\nu}\right)\left(\partial_{x}\right) \leq C_{8} e^{M_{8} i}$, for some constant $C_{8}, M_{8}>0$. It follows that

$$
\mid L_{\partial_{x}}\left(\theta_{\alpha_{i}} \kappa_{i}\left(\mathbf{x}^{\left(\alpha_{i}\right)}\left(E_{\mathbf{x}^{\left(\alpha_{i-1}\right)}\left(\mu_{i-1}, \tau_{i-1}\right)}^{\nu} \cdots E_{\mathbf{x}^{\left(\alpha_{1}\right)}\left(\mu_{1}, \tau_{1}\right)}^{\nu}(x), \mu_{i}, \tau_{i}\right)\right)|\leq| d \theta_{\alpha_{i}} \kappa_{i}|| \partial_{x} \mid C_{8} e^{M_{8} i} .\right.
$$

We turn to the derivatives of $\hat{\kappa}(a):=\kappa_{0}\left(a\left(\mathbf{x}^{\left(\alpha_{k}\right)}\left(\mu_{k}, \tau_{k}\right) \cdots \mathbf{x}^{\left(\alpha_{1}\right)}\left(\mu_{1}, \tau_{1}\right)(x)\right)^{-1}\right)$ on some exponential coordinates patch $\mathbf{x}^{(\beta)}$. One has

$$
\begin{aligned}
& \hat{\kappa}\left(\mathbf{x}^{(\beta)}(x, \mu, \tau)\right) \\
& \quad=\kappa\left(\mathbf{x}^{(\beta)}(\mu, \tau) \hat{\mathbf{x}}^{\left(\alpha_{k}\right)}\left(\mu_{k}, \tau_{k}\right) \cdots \hat{\mathbf{x}}^{\left(\alpha_{1}\right)}\left(\mu_{1}, \tau_{1}\right)\left(E_{\mathbf{x}^{\left(\alpha_{i-1}\right)}\left(\mu_{i-1}, \tau_{i-1}\right)}^{\nu} \cdots E_{\mathbf{x}^{\left(\alpha_{1}\right)}\left(\mu_{1}, \tau_{1}\right)}^{\nu}(x)\right),\right.
\end{aligned}
$$

where $\hat{\mathbf{x}}^{\left(\alpha_{i}\right)}(\mu, \tau):=\exp _{Z_{1}} \cdots \exp _{Z_{|I|}} \exp _{\mu_{1} X_{1}^{\left(\alpha_{i}\right)}} \cdots \exp _{\mu_{p} X_{p}^{\left(\alpha_{i}\right)}} \exp _{\tau_{1} Y_{1}} \cdots \exp _{\tau_{q} Y_{q}}$. Suppose further that $x \in B\left(r e^{-k(H+(|I|+p+q) \omega)}, \mathrm{M}_{1}\right)$, then

$$
E_{\mathbf{x}^{\left(\alpha_{i-1}\right)}\left(\mu_{i-1}, \tau_{i-1}\right)}^{\nu} \cdots E_{\mathbf{x}^{\left(\alpha_{1}\right)}\left(\mu_{1}, \tau_{1}\right)}^{\nu}(x) \in B\left(r e^{-k H}, \mathrm{M}_{1}\right)
$$

and from Theorem 2.14, one can write:

$$
\hat{\kappa}\left(\mathbf{x}^{(\beta)}(x, \mu, \tau)\right)=\kappa\left(\mathbf{x}^{\left(\beta^{\prime}\right)}\left(E_{\mathbf{x}^{\left(\alpha_{i-1}\right)}\left(\mu_{i-1}, \tau_{i-1}\right)}^{\nu} \cdots E_{\mathbf{x}^{\left(\alpha_{1}\right)}\left(\mu_{1}, \tau_{1}\right)}^{\nu}\right)(x), \varphi(x, \mu, \tau), \psi(x, \mu, \tau)\right) .
$$

Note that since $\kappa_{0}$ is supported on $U^{0}$, the right hand side vanishes if $\|\tau\|>k H_{1}$ for some $H_{1}$. Moreover, by the second part of Theorem 2.14, one has the estimate

$$
\left|L_{\partial_{x}} \hat{\kappa}\left(\mathbf{x}^{(\beta)}(x, \mu, \tau)\right)\right| \leq C_{9} e^{M_{9} k}\left|d \kappa_{0}\right| .
$$

In particular, put

$$
\begin{aligned}
\kappa_{0}(a) & :=\chi\left(2 r^{-1} \mathbf{s}^{*} \rho(a)\right) G_{N}(a), \\
\kappa_{i}(a) & :=\chi\left(2 r^{-1} \mathbf{s}^{*} \rho(a)\right) R_{N}(a), \quad i \leq k-1, \\
\kappa_{k}(a) & :=\chi\left(2 r^{-1} H^{k} \mathbf{s}^{*} \rho(a)\right) R_{N}(a) .
\end{aligned}
$$

Differentiating under the integral sign and summing over all $\alpha_{i}$, we conclude that

Lemma 3.2. One has the uniform estimate

$$
\begin{aligned}
& \mid \partial_{x}\left(\chi\left(2 r^{-1} \mathbf{s}^{*} \rho\right) G_{N} \circ \chi\left(2 r^{-1} \mathbf{s}^{*} \rho\right) R_{N} \circ \cdots \circ \chi\left(2 r^{-1} \mathbf{s}^{*} \rho\right) R_{N}\right. \\
&\left.\circ \chi\left(2 r^{-1} H^{k} \mathbf{s}^{*} \rho\right) R_{N}\right)\left(\mathbf{x}^{(\beta)}(x, \mu, \tau)\right) \mid \leq C_{10} e^{M_{10} k},
\end{aligned}
$$


for some $C_{10}, M_{10}$ (note that $M_{8}$ is independent of $\left.s, N\right)$. Moreover the same estimate holds for the $\partial_{\tau_{j}}$ and $\partial_{\mu_{j^{\prime}}}$ derivatives.

3.3. $C^{1}$ estimation away from $\mathcal{G}_{1}$. In the following, we make the additional assumption that $\mathcal{G}$ is non-degenerate or uniformly degenerate. Denote by $\iota: \mathrm{M}_{0} \times \mathrm{M}_{0} \rightarrow$ $\mathcal{G}$ the embedding of the invariant sub-manifold. Since the target and source maps on $\mathrm{M}_{0} \times \mathrm{M}_{0}$ are respectively the projection onto the first and second factor, one has

$$
(\mathbf{t} \times \mathbf{s}) \circ \iota=\mathrm{id}_{\mathrm{M}_{0} \times \mathrm{M}_{0}}
$$

Using the fact that $d \mathbf{t}(\tilde{V})=\nu\left(d \mathbf{r}_{a}^{-1}(\tilde{V})\right)$, for any $\tilde{V} \in T_{a} \mathcal{G}, a \in \mathcal{G}$ satisfying $d \mathbf{s}(\tilde{V})=$ 0 , it follows that

$$
d \iota(V \oplus 0)=d \mathbf{r}_{\iota(x, y)}\left(\nu^{-1}(V)\right)
$$

for any $V \oplus 0 \in T_{(x, y)} \mathrm{M}_{0} \times \mathrm{M}_{0}$. Similarly,

$$
d \iota(0 \oplus W)=d\left(\mathbf{i} \circ \mathbf{r}_{\iota(y, x)}\right)\left(\nu^{-1}(W)\right) .
$$

It is straightforward to compute the coordinate vector fields for the exponential coordinates:

$$
\begin{aligned}
d \mathbf{t}\left(\partial_{\tau_{i}}(x, \mu, \tau)\right) & =\nu\left(E_{\tau_{q} Y_{q}} \cdots E_{\tau_{i+1} Y_{i+1}} Y_{i}\right)\left(E_{\tau_{i} Y_{i}}^{\nu} \cdots E_{\tau_{1} Y_{1}}^{\nu} E_{\mu \cdot X^{(\alpha)}}^{\nu} E_{Z_{I^{(\alpha)}}}^{\nu}(x)\right) \\
d \mathbf{t}\left(\partial_{\mu_{i^{\prime}}}(x, \mu, \tau)\right) & =\nu\left(E_{\tau \cdot Y}^{\nu} E_{\mu_{p} X_{p}^{(\alpha)}} \cdots E_{\mu_{i^{\prime}+1} X_{i^{\prime}+1}^{(\alpha)}} X_{i^{\prime}}^{(\alpha)}\right)\left(E_{\mu_{i^{\prime}} X_{i^{\prime}}^{(\alpha)}}^{\nu} \cdots E_{\mu_{1} X_{1}^{(\alpha)}}^{\nu} E_{Z_{I^{(\alpha)}}}^{\nu}(x)\right) .
\end{aligned}
$$

Similarly, one computes

$$
\begin{aligned}
& d \mathbf{t}\left(\partial_{x}(x, \mu, \tau)\right)=d\left(E_{\tau \cdot Y}^{\nu} E_{\mu \cdot X^{(\alpha)}}^{\nu} E_{Z_{I^{(\alpha)}}}^{\nu}\right)\left(\partial_{x}(x)\right) \\
& d \mathbf{s}\left(\partial_{x}(x, \mu, \tau)\right)=\partial_{x}(x)
\end{aligned}
$$

where $\partial_{x}(x)$ on the right hand side is regarded as a tangent vector on $\mathrm{M}$.

On $\mathcal{G}_{0}=\mathrm{M}_{0} \times \mathrm{M}_{0}$, one can write

$$
\left(\kappa_{0} \circ \cdots \circ \kappa_{k}\right)(a)=(\mathbf{t} \times \mathbf{s})^{*} \int \iota^{*} \kappa_{0}\left(\mathbf{t}(a), b_{1}\right)\left(\prod_{l=1}^{k-1} \iota^{*} \kappa_{l}\left(b_{l}, b_{l+1}\right)\right) \iota^{*} \kappa_{k}\left(b_{k}, \mathbf{s}(a)\right) d b_{1} \cdots d b_{k} .
$$


It is then straightforward to differentiate along the coordinate vector fields:

$$
\begin{aligned}
& \partial_{x}\left(\kappa_{0} \circ \cdots \circ \kappa_{k}\right)(a) \\
& =\int\left(L_{d E_{\tau \cdot Y}^{\nu} E_{\mu \cdot X}^{\nu(\alpha)}} E_{Z^{(\alpha)}}^{\nu} \partial_{x} \oplus 0 \iota^{*} \kappa_{0}\right)\left(\mathbf{t}(a), b_{1}\right)\left(\prod_{l=1}^{k-1} \iota^{*} \kappa_{l}\left(b_{l}, b_{l+1}\right)\right) \iota^{*} \kappa_{k}\left(b_{k}, \mathbf{s}(a)\right) d b_{1} \cdots d b_{k} \\
& +\int \iota^{*} \kappa_{0}\left(\mathbf{t}(a), b_{1}\right)\left(\prod_{l=1}^{k-1} \iota^{*} \kappa_{l}\left(b_{l}, b_{l+1}\right)\right)\left(L_{0 \oplus \partial_{x}} \iota^{*} \kappa_{k}\left(b_{k}, \mathbf{s}(a)\right)\right) d b_{1} \cdots d b_{k} \\
& \partial_{\tau_{i}}\left(\kappa_{0} \circ \cdots \circ \kappa_{k}\right)(a) \\
& =\int\left(L_{\nu\left(E_{\tau_{q} Y_{q}} \ldots E_{\tau_{i+1} Y_{i+1}} Y_{i}\right) \oplus 00^{*}} \kappa_{0}\right)\left(\mathbf{t}(a), b_{1}\right)\left(\prod_{l=1}^{k-1} \iota^{*} \kappa_{l}\left(b_{l}, b_{l+1}\right)\right) \iota^{*} \kappa\left(b_{k}, \mathbf{s}(a)\right) d b_{1} \cdots d b_{k} \\
& \partial_{\mu_{i^{\prime}}}\left(\kappa_{0} \circ \cdots \circ \kappa_{k}\right)(a)
\end{aligned}
$$

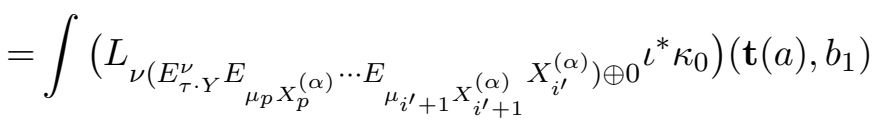

$$
\begin{aligned}
& \left(\prod_{l=1}^{k-1} \iota^{*} \kappa_{l}\left(b_{l}, b_{l+1}\right)\right) \iota^{*} \kappa\left(b_{k}, \mathbf{s}(a)\right) d b_{1} \cdots d b_{k} \text {. }
\end{aligned}
$$

Using Equation (14) and the hypothesis that $\mathcal{G}$ is non-degenerate or uniformly degenerate, we have the estimate

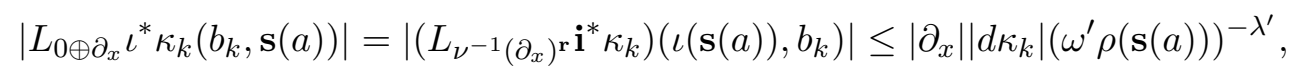

Using same arguments with $0 \oplus \partial_{x}$ replaced by $d E_{\tau \cdot Y}^{\nu} E_{\mu \cdot X^{(\alpha)}}^{\nu} E_{Z_{I^{(\alpha)}}^{\nu}} \partial_{x} \oplus 0$, one gets

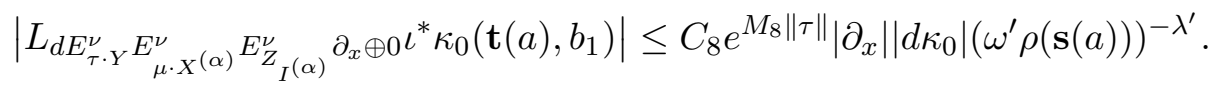

(Here we used the fact that $\left|d E_{\tau \cdot Y}^{\nu} E_{\mu \cdot X^{(\alpha)}}^{\nu} E_{Z_{I^{(\alpha)}}}^{\nu} \partial_{x}\right| \leq C_{8} e^{M_{8}\|\tau\|}\left|\partial_{x}\right|$. ) Again, put

$$
\begin{aligned}
\kappa_{0} & :=\left(1-\chi\left(2 r^{-1} \mathbf{s}^{*} \rho\right)\right) G_{N}, \text { or } \chi\left(2 r^{-1} \mathbf{s}^{*} \rho\right) G_{N}, \\
\kappa_{i} & :=\left(1-\chi\left(2 r^{-1} \mathbf{s}^{*} \rho\right)\right) G_{N}, \text { or } \chi\left(2 r^{-1} \mathbf{s}^{*} \rho\right) R_{N}, \quad i \leq k-1, \\
\kappa_{k} & :=\left(1-\chi\left(2 r^{-1} H^{k} \mathbf{s}^{*} \rho\right)\right) R_{N}, \text { or } \chi\left(2 r^{-1} H^{k} \mathbf{s}^{*} \rho\right) R_{N},
\end{aligned}
$$

but except the combination appeared in the last section. Observe that

$$
\iota^{*} \kappa_{0}\left(\mathbf{t}(a), z_{1}\right)\left(\prod_{l=1}^{k-1} \iota^{*} \kappa_{l}\left(z_{l}, z_{l+1}\right)\right) \iota^{*} \kappa_{k}\left(z_{k}, \mathbf{s}(a)\right)=0
$$

unless $\rho(\mathbf{s}(a)) \geq H^{-k} e^{-k \omega \chi_{0}}$. Together with Equations (15) and (16), one gets the uniform bound

$$
\mid L_{\partial_{x}}\left(\iota^{*} \kappa_{0}\left(\mathbf{t}\left(\mathbf{x}^{(\alpha)}(x, \mu, \tau), b_{1}\right)\left(\prod_{l=1}^{k-1} \iota^{*} \kappa_{l}\left(b_{l}, b_{l+1}\right)\right) \iota^{*} \kappa_{k}\left(b_{k}, x\right)\right) \mid \leq C_{11} e^{M_{11} k} .\right.
$$

In other words, one has

$$
\begin{array}{r}
\mid \partial_{x}\left(-\chi\left(2 r^{-1} \mathbf{s}^{*} \rho\right) G_{N} \circ \chi\left(2 r^{-1} \mathbf{s}^{*} \rho\right) R_{N} \circ \cdots \circ \chi\left(2 r^{-1} \mathbf{s}^{*} \rho\right) R_{N} \circ \chi\left(2 r^{-1} H^{k} \mathbf{s}^{*} \rho\right) R_{N}\right. \\
\left.+G_{N} \circ R_{N} \circ \cdots \circ R_{N}\right)\left(\mathbf{x}^{(\beta)}(x, \mu, \tau)\right) \mid \leq C_{12} e^{M_{12} k}
\end{array}
$$


and similar for other coordinate vector fields.

3.4. Regularity of the heat kernel. Adding Equation (17) and Lemma 3.2 together, and using the fact that the volume of the set $\Sigma \subset \mathbb{R}^{k}$ equals $(k !)^{-1} t^{k}$, it follows that the sum

$$
\sum_{k=0}^{\infty}(-1)^{k} Q_{N}^{(k)}(\cdot, t)
$$

converges uniformly and absolutely in $C^{1}$ for $N$ sufficiently large.

Clearly, similar arguments holds for all $l=1,2, \cdots$ (in particular Equation (5) can be differentiated repeatedly and estimates in the form of Lemma 2.13 still holds). Hence we conclude that

Theorem 3.3. Let $\mathcal{G}$ be a boundary groupoid of the form $\mathcal{G}=\mathrm{M}_{0} \times \mathrm{M}_{0} \bigsqcup \mathrm{M}_{1} \times$ $\mathrm{M}_{1} \times \mathbb{R}^{q}$, that is either non-degenerate or uniformly degenerate. Suppose furthermore that there exists a finite collection of exponential coordinates charts $\left\{\mathbf{x}^{(\alpha)}\right\}$ satisfying Assumption [2.9. Then the heat kernel $Q$ lies in $C^{\infty}(\mathcal{G} \times(0, \infty))$.

\section{ACKNOWLEDGEMENTS}

The author would like to thank Victor Nistor for many useful discussions. This project is supported by the AFR(Luxembourg) postdoctoral fellowship.

\section{REFERENCES}

[1] P. Albin. A renormalized index theorem for some complete asymptotically regular metrics: the Gauss-Bonnet theorem. Adv. in Maths, 213(1):1-52, 2007.

[2] N. Berline, E. Getzler, and M. Vergne. Heat kernels and Dirac operators. Springer-Verlag, 1992.

[3] R.L. Fernandes. Lie algebroids, homonomy and characteristic classes. Adv. in Maths., 170:119 179, 2002.

[4] J. L. Heitsch. Bismut super-connections and the Chern characters for Dirac operators on foliated manifolds. K-Theory, 9:507-528, 1995.

[5] K. Mackenzie. Lie groupoids and Lie algebroids in differential geometry. Cambridge University Press, 1987.

[6] R.B. Melrose. The Atiyah-Patodi-Singer index theorem. A K Peters, 1993.

[7] V. Nistor. Groupoids and integration of Lie algebroids. J. Math. Soc. Japan, 52(4):847-868, 2000.

[8] V. Nistor, A. Weinstein, and P. Xu. Pseudodifferential operators on differential groupoids. Pac. J. Maths, 189(1):117-152, 1999.

[9] B.K. So. Pseudo-differential operators, heat calculus and index theory of groupoids satisfying the Lauter-Nistor condition. PhD thesis, The University of Warwick, 2010.

[10] B.K. So. On the full calculus of pseudo-differential operators on boundary groupoids with polynomial growth. preprint, arXiv.org/abs/1111.7274, 2011. 